\title{
Resistencias y reproducciones de mujeres académicas: Estrategias de supervivencia en la academia patriarcal/neoliberal
}

Resistance and reproductions of academic women: Survival strategies in the patriarchal/neoliberal academy

\author{
Giazú Enciso Domínguez \\ University of Houston Clear Lake \\ Maite González-Yáñez \\ Universidad Adolfo Ibáñez \\ Francesca Chiappini \\ Investigadora independiente
}

\section{Resumen}

En el marco de un estudio más amplio sobre transformación de la ciencia en Chile realizamos un estudio cualitativo a través de entrevistas activas a 20 mujeres científicas que habitan la academia. En primer lugar, reconocemos que la academia se está transformando en un espacio donde la hiperproductividad, individualismo y evaluación constante, entre otros indicadores, han tomado las riendas hacia una academia neoliberal. En segundo lugar, reconocemos la academia como espacio de trabajo creado por/para hombres y, por ende, patriarcal. Nuestro objetivo es visibilizar y describir críticamente las estrategias que usan las mujeres académicas. Analizamos cuáles son sus puntos de resistencia a los sistemas neoliberal y patriarcal, pero también cómo estas estrategias pueden reproducir los mismos sistemas de los que se desean rebelar. Creamos cinco códigos que describen y analizan cada estrategia, a saber: masculinización, perfeccionismo, malabarismo, limitación y sororidad.

Palabras clave: Academia; Neoliberalismo; Patriarcado; Género

\section{Abstract}

In the framework of a broader study, in this article we carry out a qualitative study through active interviews with academic women who inhabit contemporary academia. We recognize academy is transforming in a space where hyper-productivity, individualism, and constant evaluation among other indicators have taken the reins towards a neoliberal academy. Later on, we recognize the academy as a patriarchal space. We aim to detect, make visible and critically describe the strategies used by academic women to inhabit that space. At the same time, we analyze the strategies' points of resistance to neoliberal and patriarchal systems, but also how these strategies can reproduce the very systems they want to rebel against. We create five codes that describe and critically analyze each of these strategies, namely: Masculinization, Perfectionism, Juggling, Boundaries, and Sorority. Finally, we reflect on these strategies and question the patriarchal and neoliberal logics within contemporary academia 


\section{ACADEMIA NEOLIBERAL}

La academia ha estado sumergida en una continua transformación producto de cambios en el sistema político, social y económico. Durante la conferencia mundial sobre Ciencia de la UNESCO del año 2000, diferentes organismos internacionales sugieren a los países darle prioridad a la educación superior (ES) y al desarrollo científico-tecnológico para fortalecer las economías locales.

Específicamente, Chile comienza los cambios académicos en los setenta en el contexto de las "siete modernizaciones" de Estado implementadas por el dictador Augusto Pinochet, que buscaban construir un nuevo orden neoliberal (Oliva, 2008). Estas "modernizaciones" tenían el fin de realizar cambios radicales en política laboral, seguridad social, educación, salud, descentralización regional, agricultura y el aparato judicial (Bravo, 2012).

Esta reforma es liderada por economistas como Hayek, Friedmann y Buchanan, conocidos como los Chicago boys, discípulos del economista Milton Friedmann que, en el contexto dictatorial, asumieron la autoridad política y económica (Fardella, 2020). En este contexto, Chile se convirtió en uno de los primeros países donde incorporaron nuevos mecanismos de administración de la ciencia y mecanismos propios de lógica corporativa/privada (Brunner, 2008; Slaughter y Leslie, 1997). Su objetivo fue revitalizar al Estado, apremiándolo a dejar atrás la burocracia e integrarse a la eficiencia y agilidad del mercado (Fardella et al., 2015, p. 1626).

Así, aparece la New Public Managment (NPM) (Fardella, 2013), cuyo objetivo es reorganizar el vínculo entre Universidad y Estado, promoviendo acciones como la descentralización y diversificación de las fuentes de financiamiento del trabajo científico (Oyarzún y Cornejo, 2020), incorporación de la competencia como principal mecanismo regulador, instalación de una cultura del accountability y desarrollo de sofisticados instrumentos de acreditación y medición de los procesos de trabajo (Fardella et al., 2019). Andrés Bernasconi (2009) afirma que los instrumentos dentro de la academia enfatizan la medición de los productos como, por ejemplo: números de publicaciones en revistas de alto impacto, número de búsquedas, número de descargas, número de citas..., sin embargo, estos indicadores son representaciones simplificadas de lo que significa el trabajo científico (Fardella et al, 2017). En el caso chileno, estos instrumentos vienen acompañados de incentivos económicos que premian el alcance del objetivo: por cada publicación en una revista de alto impacto, por el número de publicaciones realizadas en un margen de tiempo o por ganar un fondo de investigación siempre hay un incentivo económico. Estos estímulos pueden ser individuales, departamentales o institucionales. Hay una contraparte a estos estímulos: la facilitación de castigos por no alcanzar los objetivos. La justifica- 
ción de las penalizaciones es la mejora continua de la gestión interna, fomentar la rendición de cuentas, el control y mejorar/reducir el gasto público (Morales, 2014). Los mecanismos para administrar la ciencia dan importancia a los resultados por sobre el proceso (Tünnermann y De Souza, 2003). Investigaciones como la de Carla Fardella, Vicente Sisto y Felipe Jiménez (2017) evidencian que la fuerza de trabajo académica no está alineada con estos mecanismos, reportando diversas tensiones. Esta nueva política administrativa ha generado diversos efectos negativos y opresiones para el personal docente, incluyendo: 1) el desencuentro cultural entre los nuevos modelos de gestión y las comunidades científicas (Merton, 1973; Ordorika y Navarro, 2006; Pusser, 2008; Ramos, 2012a, 2012b); 2) las transformaciones en el proceso de trabajo científico como la aceleración de los ritmos de trabajo, propuestos por Maggie Berg y Barbara Seeber (2016), la estandarización de la producción de artículos académicos como único producto válido (Gómez et al., 2015; Santos, 2015), la hiper individualización del proceso productivo y el aumento estratégico de coautorías en la publicación científica (Menzies y Newson, 2007); y 3) las repercusiones en la salud laboral del profesorado, como estrés, desinterés y pérdida de sentido (Chandler et al., 2002; Berg y Seeber, 2016) o la intensificación de actividades y responsabilidades rebasando los límites tradicionales del espacio de trabajo (Gill, 2009).

La academia se ha convertido en un espacio donde las prácticas neoliberales dictan la agenda académica y las vidas de las personas que la habitan. Demandado un modelo laboral altamente individualizado, competitivo y autoexigente, sin límites de tiempo, espacio, energía o emoción y donde la vida personal y profesional demanda diferentes posiciones en el mundo (Fardella y Corvalán, 2020).

\section{ACADEMIA PATRIARCAL}

En 1877 se promulgó el Decreto Amunátegui que autorizaba el acceso a las mujeres a los estudios universitarios en Chile (Salinas Ibacache, 2019). No fue hasta los setenta que la presencia de la mujer fue visible en la academia. El feminismo blanco europeo de la segunda ola y los movimientos sociales lograron incorporar a la mujer como categoría de análisis, lo que ocasionó la visibilización de nuevos conceptos y categorías que subrayaron la conexión de las mujeres en la academia (Kumud, 2003). María de los Ángeles Durán (1981) señala como el sexismo en la ciencia está prácticamente en cada una de sus esferas:

La selección de los temas de investigación, la selección de las perspectivas teóricas, la aceptación de los resultados de la investigación, la elección de los criterios de evaluación, la difusión de los resultados, el reconocimiento 
profesional y académico, las normas y prácticas sociales de los colectivos creadores de ciencia o conocimiento especializado, tales como Escuelas, Academias, Universidades, Sociedades Científicas. (p. 172)

Este sexismo no ha sido eliminado, sino que ahora se ejerce de manera sutil y contemporánea.

La llegada de la mujer a la academia no fue fácil, ya no solo porque la academia fuera/sea considerada territorio masculino, sino por el sistema patriarcal que la gobierna. Judith Lorber (2008) define patriarcado como sistema que promueve hegemonía y dominio de lo masculino sobre lo femenino, más que como un conjunto de conductas particulares de sujetos particulares. Este sistema se encuentra presente en la academia generando desigualdades y situaciones de opresión para las mujeres y otras disidencias. Además, prácticas que hacen que la participación de las mujeres en el mundo académico sea subestimada, no reconocida y marginada (Thomas, 1999, en Tsouroufli, 2018).

Siguiendo a Margot Pujal (1994), el análisis de las desigualdades de género en la academia no puede ir separado del análisis de los supuestos de la ciencia moderna y sus formas de conocer. Históricamente, la ciencia ha privilegiado los discursos de subjetividades pensadas como universales y legítimas en sí mismas, dejando atrás discursos de otras, por considerarlas particulares y no universales (Pujal, 1994). La ciencia moderna opera con dicotomías jerárquicas tales como público-privado, impersonal-personal, razón-emoción, abstracciónconcreción, instrumental-afectivo y masculino-femenino, asociando el primero de los términos de cada dicotomía a la subjetividad universal privilegiada en la ciencia (Enciso y Lara, 2014; Pujal, 1994). La ciencia moderna presente en la academia propone al hombre como la subjetividad privilegiada, la voz impersonal, objetiva, racional y neutral al producir conocimiento, mientras que piensa a la mujer (y otras disidencias) como voz particular, no legítima, por estar asociada a lo emocional, afectivo, personal y privado (Enciso y Lara, 2014; Pujal, 1994). De esta manera, las mujeres (y otras disidencias) quedan en una posición inferior en la academia.

Esta desigualdad epistemológica es el origen de la desigualdad entre hombres y mujeres ( $y$ de toda subjetividad que no esté alineada con lo masculino) dentro de la academia. Estos supuestos epistemológicos que subyacen a las prácticas y discursos se reproducen y perpetúan, generando situaciones de opresión para las mujeres (y otras disidencias), haciendo de la academia un lugar hostil.

Si bien se han realizado intentos por solucionar esta desigualdad, los resultados han sido superficiales. Ejemplo de ello es la estrategia que analiza Londa Schiebinger (2008) respecto a aumentar el número de mujeres dentro de la 
educación superior, táctica que Schiebinger llama Fix the numbers (2008). Estudios como los de Jacob Blickenstaff (2005) y Manfriedi et al. (2014) demuestran que existen desigualdades de género que bloquean -en todos los sentidos- las carreras de las académicas y que la estrategia de incorporar numéricamente a las mujeres a la academia no tiene sentido alguno. Hall and Sandler (1982, en Britton, 2017) acuñan el término chilly climate, para referirse a los patrones de tratamiento desigual que, a medida que se acumulan, inhiben la confianza, la autoestima y el logro de las mujeres. Por su parte Christine Heward (1994), y Bruce Macfarlane y Damon Burg (2019) trabajan el concepto de glass ceiling, que describe cómo las mujeres ven la posibilidad de ascender en sus trabajos, pero enfrentan barreras "invisibles" que les impiden lograrlo. Estas tácticas han sido ampliamente documentadas por diversas autoras acuñando diferentes conceptos para describirlas, sin embargo, poco se ha problematizado sus funciones desde una perspectiva de género dentro de la academia.

Otro concepto es leaky pipeline, como Fran Amery et al. (2015) plantean: a medida que avanza la carrera académica el número de mujeres disminuye. Durante el 2016, a nivel mundial un $53 \%$ de las mujeres se graduó de la universidad, $53 \%$ de magíster, $43 \%$ de doctorado, pero sólo $28 \%$ se dedica a la investigación (UNESCO Institute for Statistics, 2016, en UNESCO, 2017). La academia patriarcal y sus características se materializan en una brecha de género que se puede apreciar a nivel global. Según el último informe de Mujeres en Ciencia de la UNESCO (2019), las mujeres son solo el 29,3\% del personal investigador a nivel mundial; en Chile, un 31,1 \% del total. Solo un tercio de las personas académicas dedicadas a la investigación son mujeres.

Según el Informe del personal académico del Sistema de Información de Educación Superior (SIES, 2021), la participación de mujeres en educación superior ha incrementado de un 43,3\% a 44,5\% del total entre el 2016-2020. Si bien este dato puede parecer alentador, es importante tener en cuenta que este porcentaje no representa solo a las mujeres que realizan investigación (UNESCO, 2019), sino que incorpora a mujeres con funciones puramente administrativas o docentes. Además, este dato no es cruzado con información respecto a la relación laboral contractual que mantienen estas académicas con las instituciones universitarias (tiempo completo/parcial). Hay solo un $30 \%$ de mujeres académicas contratadas a tiempo completo (SIES, 2018). En cuanto a proyectos de investigación adjudicados al personal investigador, los números son aún más agudos: $28,8 \%$ de investigadoras a nivel mundial (UNESCO, 2017), que se acentúa si tomamos en cuenta que las mujeres líderes de proyectos son el 16\% (CONICYT, 2018). 
Siguiendo a Schiebinger (2008), hay otras medidas intermedias orientadas a eliminar barreras estructurales/culturales para el ascenso y supervivencia de las mujeres dentro de la academia: Fix the Institution, pero que tampoco logran por completo su objetivo. Thamar Heijstra et al. (2016) acuñan el concepto academic housework que hace referencia a la práctica que asigna a las académicas a adoptar responsabilidades asociadas a los roles de género predeterminados por la sociedad dentro del espacio de trabajo. Las mujeres ya están dentro de la academia, pero la institución continúa saboteando su presencia asignando trabajos de cuidado generando que coloquen su tiempo/energía en tareas poco valoradas dentro de la academia: trabajo administrativo, formación de personal investigador, docencia o inspirar a nuevas generaciones. Esto impide y/o hace aún más lenta la posibilidad de promoción (Macfarlane y Burg, 2019).

Finalmente, acuñamos una táctica más, que denominamos fraternidad académica o academic brotherhood y refiere a las redes compuestas por académicos hombres, entre académicos hombres, para beneficiar y recompensar a académicos hombres. De esta hermandad de hombres surgen ciertos beneficios para quienes la componen, como contrataciones, ideas de artículos, proyectos, conferencias entre otros. La fraternidad académica deja fuera a las académicas mujeres en diferentes niveles y situaciones laborales. Esta estrategia afecta la carrera de la mujer académica al inicio (contratación) durante (retención y promoción) y después (visibilización).

\section{METOdOLOGÍA}

La metodología del trabajo se sitúa bajo el paradigma cualitativo, cabe resaltar que hay un número limitado de estudios empíricos que discuten la relación entre la educación superior y NPM, por lo que nuestra contribución de recogida de experiencias de las mujeres académicas es clave para entender narrativas y experiencias de investigadoras en la contemporaneidad. Los datos reportados en este trabajo se obtuvieron entre el 2016-2018, en el marco de un estudio más amplio sobre transformación de ciencia en Chile (Fardella et al., 2017). Las participantes fueron seleccionadas por medio de un muestreo no probabilístico de snowball, cuidando la diversidad disciplinar. Las participantes son 20 mujeres científicas de la zona central chilena, con productividad académica, fondos de investigación financiados por organismos externos, trayectorias científicas diversas y pertenecen a diferentes áreas del conocimiento (Ingeniería comercial, sociología, física, educación de párvulo, educación especial, antropología, psicología, química ambiental, filosofía, ingeniería civil, educación básica, trabajo social, filosofía, derecho e historia). 
Se realizaron entrevistas activas, definidas según Norman Denzin (2001) como una interacción en la cual las participantes (entrevistador/a y entrevistado/a) son asumidas como sujetos que interactúan abiertamente, aunque guiadas por una pauta flexible con formato de respuesta abierta. La flexibilidad y espontaneidad permite a la entrevistadora ser sujeto activo y co-producir conocimiento con la persona entrevistada (Holstein y Gubrium, 1995), llevándolas a espacios discursivos nuevos, (Denzin y Lincoln, 2003, Biron, 2014). La entrevista activa se organizó en torno a tres temas específicos: trayectorias científicas, prácticas y experiencias en la producción científica e identidad laboral.

Para alcanzar profundidad/intersubjetividad en el análisis, se realizó en diversas instancias temporales. Etapa uno: el corpus de datos se codificó y analizó con base en su contenido, haciendo uso de la teoría fundamentada (Glaser y Straus,1967). Etapa dos: se crearon unidades de significado que el equipo investigador categorizó, filtró y reorganizó a partir de los objetivos de la investigación. Se obtuvo un libro de 47 códigos que en su conjunto dan cuenta de diferentes aspectos del trabajo científico de las mujeres en Chile: trayectorias científicas, prácticas, experiencias en la producción científica, identidad laboral... Etapa tres: lectura sistemática de los códigos y contexto, en la que se seleccionaron categorías que daban cuenta de estrategias que las mujeres entrevistadas utilizan para habitar y sobrevivir a la academia. Presentamos cinco categorías que describen y analizan críticamente cada una de esas estrategias, a saber: masculinización, perfeccionismo, malabarismo, limitación y sororidad.

Con el fin de salvaguardar la confidencialidad de nuestras participantes, hemos elegido pseudónimos, en este caso, nombres de piratas mujeres. Así como las piratas navegaban en los mares más peligrosos y sobrevivían (Appleby, 2013), así nuestras académicas cada día sortean un nuevo obstáculo y se reinventan. Se valen de muchas estrategias para sobrevivir, se visten de hombres (Cordingly, 1996; Stanley, 1995), se rodean de otras mujeres, trabajan el doble o el triple para asegurar su supervivencia o promoción y consiguen habitar los mares de la academia. Al igual que las académicas, a las piratas la historia patriarcal las ha dejado fuera o, en el mejor de los casos, invisibles (MacAlpine, 2016). No son citadas en los libros de historia, ni son tan famosas/reconocidas y, cuando se habla de ellas, su papel se reduce al de una meretriz (Stanley, 1995). En este artículo, rendimos homenaje a las mujeres piratas, con agencia, activas, a través de la visibilización al nombrarlas. No queremos con esto elogiar las formas de regulación, producción o explotación social que exigen a las mujeres académicas, es decir, el ser activas y responsables de sus quehaceres académicos a nivel individual, sino destacar que, pese a las lógicas patriarcales y neoli- 
berales que operan en la academia, ellas han logrado hallar estrategias que les permiten habitar la academia.

\section{ANÁLISIS}

El objetivo de este análisis es visibilizar las estrategias de las mujeres que habitan la academia y analizar críticamente cada estrategia. Describiremos cada estrategia -masculinización, perfeccionismo, malabarismo, limitar y sororidad- y las analizaremos tomando en cuenta dos elementos: resistencia y reproducción. Examinaremos cómo cada estrategia, a la vez que resiste a lógicas patriarcales y/o neoliberales, también puede reproducir otras dimensiones del sistema. A través de la problematización de las estrategias, colocamos el foco de atención en denunciar las opresiones y lógicas neoliberales y patriarcales, ubicando la responsabilidad en los sistemas y no en las personas que performan dichas estrategias.

No pretendemos penalizar a las académicas que hacen uso de las estrategias, sino reflejar la presión del sistema y las opciones que las mujeres crean para enfrentarse a una estructura que las oprime y obliga a desarrollar diferentes alternativas para sobrevivir. Al visibilizar las opresiones del sistema también vislumbramos los esfuerzos que han hecho y hacen las académicas al resistir a la idea de una academia nacida desde el patriarcado y con lógicas del neoliberalismo. Si bien somos críticas de las lógicas patriarcales y neoliberales que reproducen algunas de estas estrategias, también valoramos el esfuerzo que por años llevan haciendo las mujeres para poder formar parte de la academia.

\section{Masculinización}

Una de las estrategias más antiguas del mundo: enfundarse en los ropajes masculinos, aunque en este caso sean metafóricos. masculinización consiste en incorporar características asociadas al rol de género tradicional masculino para continuar su trayectoria científica. Las áreas en las que las mujeres se vuelven masculinas son variadas. Algunas de ellas son: vestimenta, actitudes, temas de estudio o especialización, formas y horarios de trabajo, formas de argumentar, entre otras. Esta estrategia ayuda a las mujeres a poder sobrevivir y avanzar en un espacio que no las valida como productoras de conocimiento (Pujal, 1994).

Anne Dieu le Veut nos relata cómo su trabajo en la academia lo vive como una experiencia de traducción o de travestismo:

En un sitio (la academia) en el que tienes que trabajar más en..., es como si tuvieras que traducirte en el camino. ¿Entiendes? Como explicarte en otros términos, como si tuvieras que trasvestirte un poco en, como todo sentido. (Anne Dieu le Veut, entrevista personal, julio de 2018) 
Charlotte cuenta cómo la masculinización, en su caso, tuvo que ver con sus formas de argumentación y con sus temas de estudio:

Lo que le comentaba a mis estudiantes, cuando me decían a mí que yo pensaba como un hombre. Era siempre un piropo. ¿Me entiendes? Porque me estaban diciendo con eso que era muy seria que podía argumentar bien, o sea, ¿cosas que ahora son impensables decir no? Pero que hace veinte años a mí me los decían y yo me sentía bien ¿entiendes? 0 sea, no era como que me encontraron estupidez no, yo me sentía halagada: con ese piropo. (Charlotte Badger, entrevista personal, junio de 2016)

Observamos cómo la masculinización no es siempre realizada con conciencia ni es vivida con pesar. Actuar como hombre en la academia permite sobrevivir y vivir bien, es bien visto y reforzado por el entorno. Actuar como hombre posiciona a Charlotte como persona seria/racional, alejándose de lo femenino/emocional/afectivo. De esta manera, la acerca al "sujeto ideal" de producción de conocimiento: el Hombre (Pujal, 1994), y le permite habitar la academia.

Podemos observar en las siguientes citas que la masculinización permite a las académicas sobrellevar la inseguridad y sentirse validadas. Charlotte nos cuenta cómo el escoger temas "oscuros y difíciles" de filósofos hombres y que seguían cierto modelo tradicional y masculino de filosofía, la validada:

Yo creo que el tema del género se muestra en otras cosas, en los temas que elegí por ejemplo, que son temas clásicos de filosofía masculina, filosofía alemana, cierto enfoque particular, o sea, no iba a pensar en el cuerpo, en el género porque eran poco serias en ese tiempo ¿entiendes? porque había un modelo. El doctorado fue de Heidegger y la tesis de pregrado era de Kant, entonces, claro, no se me ocurre, por ejemplo, tomar a Hanna Arendt, no era una filósofa que uno podía tomar en serio ¿me entiendes? (Charlotte Badger, entrevista personal, junio de 2016)

Entonces creo que de ahí viene el tema que, claro, la racionalidad es muy masculina y ciertos temas que uno tiene que trabajar si uno quiere ser serio en filosofía. Creo que eso marcó ciertos temas que yo empecé a estudiar filosóficamente, que eran temas tremendamente oscuros y difíciles $\mathrm{y}$, por lo tanto, eran los que yo quería porque me hacían validarme. (Charlotte Badger, entrevista personal, junio del 2016)

La masculinización también modifica las formas y horarios de trabajo. Rachel nos relata que antes de ser madre, podía realizar un "simulacro de comportamiento" masculino que le permitía trabajar como hombre. Al no tener responsabilidades fuera de su trabajo podía centrarse por completo en él: 
No había fricción porque yo podía hacer un simulacro de comportamiento, no había fricción porque yo no tenía entre que decidir, no había fricción porque no había con qué tensionar ese rol masculino que me había inventado en la pega (trabajo), podía trabajar como un hombre. Podía trabajar como un hombre, sin horario, sin tener obligación de cuidar a nadie, sin querer cuidar a nadie, podía hacerlo porque no se me presentaba ninguna tensión, a lo más me cansaba, pero daba lo mismo. (Rachel Wall, entrevista personal, mayo de 2018)

La academia como organización tiene como sujeto ideal al hombre no sólo por razones epistemológicas (Pujal, 1994), también porque ellos suelen tener pocas/ninguna responsabilidad fuera de su trabajo (Acker, 1990), esto les permite estar centrados en él, trabajar fuera de horario y que la consecuencia solo sea cansarse. Esta característica tradicionalmente masculina pueden adoptarla las mujeres académicas, pero cuando tiene a alguna persona bajo su cuidado, adoptar esta estrategia se pone cuesta arriba.

La masculinización permite a las mujeres habitar la academia mediante estrategias de resistencia, pero también hay elementos que reproduce, a continuación, describimos las tensiones de esta estrategia. La masculinización resiste al paradigma de ciencia tradicional que dicta que los hombres son los únicos productores de conocimiento válidos, y logra que las mujeres puedan producir conocimiento dentro de la academia. Al usar esta estrategia, se reproduce la idea de que la forma en que las mujeres pueden producir conocimiento en la academia es asimilándose lo más que puedan al "sujeto ideal" de producción de conocimiento: hombre. Las mujeres académicas se parecen a las mujeres que en los siglos XIX y XX se vestían con ropas masculinas para poder participar de las fuerzas armadas y navales durante las guerras, quienes llevaban a cabo un proceso de imitación de lo masculino y no una subversión activa y consciente de los roles de género (Wheelwright, 1989).

Esta estrategia, sin embargo, logra resistir al mandato de cuidado de otros como el deber ser de las mujeres. Tradicionalmente, las mujeres realizan el trabajo de cuidado de otras personas en las familias con acciones como cocinar, hacer las compras, cuidar de las criaturas, personas enfermas y personas mayores (Elson, 2000). Esta tradición es resistida por la estrategia de masculinización.

Aun con esta resistencia, rechazando el mandato del cuidado, la estrategia de masculinización, reproduce la lógica neoliberal de que el sujeto ideal de la academia es quien puede centrarse exclusivamente en su trabajo, sin estar tensionado por responsabilidades ajenas a este, o quien puede trabajar sin límites (Fardella y Corvalán, 2020). 
Reconocemos que la estrategia de masculinización, si bien permite la entrada a la mujer a habitar la academia, también reproduce el sistema de opresión al transcribir las lógicas patriarcales y neoliberales, por lo que no resuelve el problema a largo plazo. Sin embargo, es y ha sido un mecanismo efectivo para que las mujeres ocupen la academia. Como nuestras piratas, es una estrategia histórica sobre todo entre las primeras mujeres que llegaron a un terreno desconocido y $100 \%$ masculino.

\section{Perfeccionismo}

Consiste en ser lo más perfecta posible y mostrarlo en el ámbito académico. Implica un performance sin fallos para ser mostrado y validado en la academia. Esta estrategia combate la narrativa patriarcal generalizada que las mujeres no son "adecuadas" para la academia porque tienen "fallas": son débiles, emocionales, se quejan, no pueden con la carga laboral... (Cannella y Salazar Pérez, 2012). La estrategia tiene por objetivo mostrar lo perfectas que son, lo cual les permite estar dentro de la academia.

Ah sí. Es bonito. Ahora yo, te cuento mi experiencia, mi parte. 0 sea que yo tengo que llegar blindada por todas partes. Como que, a mí, como esa manera de escribir y además porque no sólo debe ser técnicamente correcto, además bonito ¿me entiendes? Perfecto. (Zheng Shi, entrevista personal, junio de 2016)

La estrategia del perfeccionismo no solo se trata de escribir bien (técnicamente), sino que escribe bonito. No deja margen a la mujer para fallar. Sin embargo, su presencia dentro de la academia, además de resistir, también contribuye con la idea de que solo hay una forma de hacer academia, que sería desde la perfección definida desde una perspectiva patriarcal.

Esta estrategia no es temporal, se ejerce a largo plazo y trae consecuencias para quien la ejerce:

Pero (las mujeres) se demoraban muchísimo en terminar sus tesis (.) seis años. $Y$ eran mujeres brillantes a mi juicio, muy inseguras porque siempre tratando de, ya, mejor, más perfecto. Yo creo que las mujeres padecen de un perfeccionismo, pero crónico, mientras que los hombres no. $Y$ el perfeccionismo crónico es bien auto flagelante en realidad, porque, es esto de que quede mejor $\mathrm{y}, \mathrm{y}$ a costa de que saben que las que están quedando mal con cada mejoramiento son ellas mismas. (Charlotte Badger, entrevista personal, junio de 2016)

La estrategia de perfeccionismo tiene un precio, como dicta el sistema neoliberal, a nivel individual. Apreciamos la tensión entre luchar por la perfección para encajar y ganar su posición en la academia al entregar calidad en su trabajo. 
Revela cómo el perfeccionismo es la estrategia para combatir los sentimientos de inseguridad y solucionar el cuestionamiento sobre si las mujeres pertenecen o no a la academia. Visualiza cómo la misma estrategia es auto flagelante pues, al buscar esa perfección, regresa el sentimiento de inseguridad sobre si el trabajo que se realiza llegó o no a la perfección.

Entonces para mí, yo me he dado cuenta para mí (con una retórica específica). Para mí tiene que ver con que esa forma de escribir me da seguridad. yo creo que ese perfeccionismo ponte tú, pero yo, en mi trayectoria yo en mi trayectoria lo asocio a la inseguridad. (Zheng Shi, entrevista personal, junio de 2016)

Finalmente, el perfeccionismo también sirve al sistema neoliberal que enfatiza la responsabilidad del individuo, exalta sus iniciativas personales y no tiene una crítica como sistema preguntándose por el tipo de sujeto que él mismo está produciendo en la sociedad.

\section{Malabarismo}

Con la estrategia del malabarismo puede ilustrarse el balanceo de responsabilidades que realizan las académicas para cumplir las exigencias tanto de la academia como las del mandato patriarcal. Implica realizar más de una tarea al mismo tiempo, ya sean tareas académicas y/o del hogar, hallando el balance. Muchas veces es vista como una cualidad "positiva" "propia de las mujeres" de la que incluso se hace alarde:

Permanente, ¿sabes cómo me siento? como estos monos de los circos que tienen, así como once platillos dando vuelta, como que ese es mi metáfora de la conciliación. Como de, como correr para que todo esté girando en su eje permanentemente. (Rachel Wall, entrevista personal, mayo de 2018)

Rachel debe mantener girando los platillos del sistema de producción neoliberal de la academia, así como también mantener girando los platillos del hogar.

Anna da cuenta cómo vive el estrés de mantener su acto de malabarismo del sistema de producción neoliberal de la academia:

Bueno, el estrés (riendo) el estrés permanente uh, te diría que esta semana es mejor que la anterior, no todas las semanas son iguales, no, claramente no, la semana pasada, eh el impacto que tiene en mí es que, cuando estoy con mucho estrés eh, he estado como tres años con mucho estrés, la verdad, porque con un proyecto de investigación que tuve con CONICYT, no había tenido productividad, no había tenido una publicación y estaba en deuda con ellos, y eso me tenía con mucho estrés, eh internamente también tengo que cumplir estas exigencias de mi escuela. (Anna Bonny, entrevista personal, octubre de 2016) 
Anna se mantiene en un estrés constante para mantenerse en la academia, además vive esta autoexplotación con culpa, se siente "en deuda". Todo esto le suma dificultad a la ya difícil tarea de los malabares.

Otro ejemplo que aborda el estrés en el malabarismo lo presenta Rachel, quien da cuenta de cómo la carga laboral le impide poder cumplir con las responsabilidades de cuidado de otras personas, que los roles de género le asignan, y cómo la estrategia también implica culpa:

Claro ahí es cuando digo, "puta la wea, la servilleta, llevamos una semana limpiándonos el poto con servilletas, loco, porque no he ido al supermercado, cómo no soy capaz de acordarme de que hay que ir a comprar confort", ¿por qué? porque lo dai todo en la pega. (Rachel Wall, entrevista personal, mayo de 2018)

La Malabarista resiste a la idea que sostiene que la academia no es un lugar para ellas, al mismo tiempo les "permite" "conciliar", si es que así se le puede llamar, su trabajo con el rol de cuidado que deben cumplir en sus casas. La estrategia de malabarismo les permite sobrevivir en la academia y no renunciar por completo a su rol (de género) de cuidadora, resistiendo además al ideal de trabajador masculino.

En esta estrategia, se presentan formas de reproducción tanto a lógicas neoliberales como patriarcales. El malabarismo reproduce los roles tradicionales de género, ya que retira la presión y/o responsabilidad de las parejas hombres o padres de cuidar de otros, bajo la excusa de que ellos "no pueden hacer eso" y "tienen que estar en una sola cosa":

No eeh si yo tengo algo que entregar... yo sé que los niños están con él y no me preocupo y viceversa... eh... nos dividimos las tareas... entonces yo estoy a cargo de comida y salud y él está a cargo de mantención de la casa... bueno mantención del perro... y... no me acuerdo que otra cosa... dentista (risas) así es... o sea, si hay dentista yo me despreocupo y si él está a cargo de las idas al dentista, y si alguien se enferma... yo soy la que los llevo y compro los remedios... etc., y.... lo elegimos por las cosas que nos gustan... a mí me gusta ir al supermercado entonces yo soy la que me encargo de eso... pero no... no siento... como... que por ser mujer tengo una carga. (Anna Bonny, entrevista personal, octubre de 2016)

En esta cita podemos ver la división clara y desequilibrada del trabajo en casa. Anna admite que se encarga de: (1) las compras, (2) la manutención de la casa, (3) la comida y (4) la salud, mientras que su pareja se ocupa del (1) perro y (2) dentista. La carga es claramente dispar, pero hay una negación de ello. Como describe en la primera parte de la cita, está "contra la pared" pero también hay una negación al respecto, pues comenta que hay "apoyo mutuo". 
Podemos observar cómo la estrategia del malabarismo reproduce las lógicas patriarcales y las lógicas neoliberales de producción académica por medio de la auto explotación de quienes la usan. Esta estrategia le permite permanecer en la academia, a costa de responsabilizarse de su acto de malabarismo entre la casa y el trabajo.

\section{Limitación}

Esta estrategia hace énfasis en cómo las mujeres académicas deben poner límites y aprender a decir que no en su vida laboral. Marcela Lagarde explica cómo las mujeres estamos construidas como seres de amor (2001), argumenta que la mujer ha sido educada para complacer al otro, por lo que negarse a ello le es difícil. Lai Coi San dice:

Yo creo que a las mujeres como les cuesta decir no. Y es difícil hablar sobre el tema de género con mujeres, como: género sin estereotipos ¿no? Pero yo creo que hay una tendencia de querer complacer como a que todas queden bien ¿no? (Lai Coi San, entrevista personal, junio de 2018)

Siendo profesionistas mujeres, no son per se leídas como mujeres "ocupadas" que "no hay que interrumpir", con un trabajo "serio"; su identidad de mujer, los roles tradicionales de género tienen más peso y pareciera ser que las personas pueden hacer uso de sus tiempos:

$\mathrm{Si}$, si, si... o sea, para la organización es clave, entonces a veces claro, vienen mis ayudantes y tengo un ayudante este semestre que es súper regalón y quiere conversar y, hoy en la mañana le dije: oye sabes que, ándate le dije, te tengo que echar porque viene una chica a las tres, que le voy a dar una entrevista, tengo que corregir pruebas y tengo una reunión de almuerzo así es que... eh... a veces va por ahí ¿no? A ponerle límites porque hay múltiples exigencias. (Anna Bonny, entrevista personal, octubre de 2016)

El poner límites resiste así al sistema patriarcal. Sin embargo, podemos ver cómo esta estrategia tiene varias aristas. Analizando el sistema neoliberal vemos una paradoja: poner los límites puede restringir la cantidad de trabajo o elegir entre las diferentes actividades y con ello bloquear algunas prácticas neoliberales en cuanto a la hiperproductividad, pero también permite una organización alrededor del trabajo para sacar adelante esa misma productividad "que le voy a dar una entrevista, tengo que corregir pruebas y tengo una reunión de almuerzo".

Interesante, es el costo emocional que tiene esta estrategia. El no poner límites implica sentirse culpables de no hacer bien el trabajo, de no organizarse 
eficientemente. El poner los límites también implica culpa por no hacerse cargo de todas las tareas.

Sí, yo creo que hay muchas ventajas, bueno, como lo que sí hay es una sobrecarga de trabajo continuamente, pero yo creo que ahí también somos culpables porque hay que poner límites, me ayudó a hablar contigo porque siempre ayuda la opinión de un tercero y quizás tengo que decir no, no puedo decir que todo sí, porque no porque no puedo hacerlo como a mí me gustaría hacerlo. (Charlotte de Berry, entrevista personal, junio de 2016)

Vemos que Charlotte es consciente de la sobrecarga del trabajo y de la respuesta ante esa práctica que es decir no. Es interesante leer que el "no" viene dado porque no puede "hacerlo como a ella le gustaría". Aunque es leído como un exceso de productividad, el "no", no es a realizar el trabajo, sino a no poder hacer el trabajo bien hecho. Vemos entretejida la estrategia del perfeccionismo para habitar la academia, una académica ciertamente preferiría decir que no, antes que entregar un producto imperfecto.

Finalmente, vemos que estos límites son flexibles y muchas veces no se colocan a tiempo o con suficiente margen de acción. Las académicas suelen tener un problema al poner sus límites porque la acompañan de una narrativa de privilegio, donde no tiene derecho a quejarse dada su posición en el mundo

Yo creo que todos quienes tenemos un trabajo académico pagado y con un contrato, eso implica pago de licencia médica, vacaciones, somos unos privilegiados (...) yo lo único que tengo que cumplir es cumplir mis publicaciones, publicar en la universidad, hacer bien docencia pero uno es su propio jefe, es un administrador de su propia fuerza laboral, no somos pobres. (Charlotte de Berry, entrevista personal, junio de 2016)

Esta estrategia reproduce los objetivos del sistema neoliberal al poner el peso de la "solución" en ella. El sistema no da opciones para limitar la cantidad de trabajo y ella debe hacerse responsable de poner límites. Aunado a la construcción de género de la mujer y la culpa, hace esto una tarea muy difícil. Si la académica pone límites, el sistema académico y sus políticas neoliberales, hiper-productivas e hiper-individualistas gana, porque la solución vino de ella como individuo y no del sistema. Si la académica no pone límites, el sistema gana también pues ella seguirá produciendo para la academia.

\section{Sororidad}

La palabra sororidad viene del francés soeur que significa hermana. Es una palabra que se ha rescatado por colectivos feministas para hablar de la hermandad entre mujeres y el apoyo, para combatir la narrativa de rivalidad que hemos aprendido (Lagarde, 2009). Esta estrategia consiste en formar redes de 
mujeres que se ayudan a salir adelante en un espacio productivo, patriarcal y neoliberal, desde el apoyo emocional y de conciliación trabajo/familia a la forma de producción del conocimiento.

Yo ¿sabes qué? he entendido, ahora también, post mamá, que hay una cosa de empatía es muy heavy. (¿En las mujeres?) En las mujeres mamás, o sea, a mí, si una mina(mujer) me dice "weona, estoy tratando de coordinar los hijos, el colegio, la casa, el supermercado, dime a qué hora puedes venir", "a las cinco", "ya, vale a las cinco" me adecúo ¿sabes?, o sea, si yo no estoy con todos los problemas en ese minuto de ese tipo, y la chica necesita que estar a las cinco, voy a las cinco, no a las cinco uno, a las cinco, y un hombre va a llegar a las cinco y media, porque no valora de la misma manera el tiempo. (Rachel Wall, entrevista personal, mayo de 2018)

Esta estrategia busca resistir a lógicas patriarcales, que hacen alusión a que las mujeres no deben participar del espacio productivo fuera de la casa, por lo que las académicas crean redes de apoyo para la conciliación trabajo familia y para empatizar y gestionar las labores académicas productivas; por ende, habitar la academia.

Entonces, las mujeres tenemos redes en la amistad, y nos apoyamos en la amistad, en las dificultades, en las penas, en las (risas) ¿cachai? Como que las mujeres, yo creo, que somos mejores en el trabajo yo creo que no sé cuánto nos apoyamos, digamos, o nos apoyamos más en lo emocional. (Sister Ping, entrevista personal, junio de 2018)

La academia patriarcal ciertamente no incluye a las mujeres en su identidad de madres académicas, no las contempla. Si el sistema no lo hace, ellas se organizan de manera independiente para ser incluidas. Claramente las llamadas políticas de conciliación no terminan de entender la trascendencia del fenómeno mujer y mucho menos el de ser madres, por lo que estas redes no están institucionalizadas. Esta estrategia es individual y pone una vez más el peso en ellas para la búsqueda de soluciones y no en el sistema académico. Cabe resaltar la necesidad de ampliar los estudios que escriban específicamente sobre madres académicas y sus estrategias, esa intersección invisibilizada e ignorada por la academia.

Sí, otro aspecto por ejemplo que, que... requiere de coordinación a veces, cuando tengo que viajar por ejemplo, si tengo que ir a una conferencia... claro ahí... requiere de toda una red de... de apoyo... de más que nada un apoyo emocional eh... cuando... yo voy a viajar tengo la suerte que mi suegra vive muy cerca de mi casa entonces siempre se va a mi casa cuando yo no estoy entonces las niñas están con la abuela. (Anna Bonny, entrevista personal, octubre 2016) 
Y digo “pucha, están mis hijas y, bueno yo tengo, mi mamá me ayuda en todo, o sea, o si no yo no podría hacer nada en realidad poh, mi mamá ya ahí se queda los martes y los jueves con mi hijo (...) una mujer que no tiene la ayuda de la mamá, estás jodida no puedes hacer nada pues, entonces más te estresas, y así empieza ese círculo de la culpa. (Sister Ping, entrevista personal, junio de 2018)

Estas redes no solo actúan en el exterior de la academia, sino al interior. Buscan resistir a lógicas neoliberales que fomentan la competencia y premian el desempeño individual, de modo que buscan colectivizar el espacio productivo al mismo tiempo resisten a la hacer academia desde una perspectiva masculina. Las académicas buscan una dinámica no competitiva o destructiva sino generosa:

Las mujeres también, no era, o sea, son como más generosas en general que los hombres, (los hombres son) como más duros. Creo que en eso sí noté diferencia, o sea, las mujeres no iban a hacer preguntas que te iba a desbaratar todo. Sino que eran preguntas más de real interés o muchas veces callá, para no dañar, digamos ¿no? (...) Entonces como que hay una actitud como mucho más solidaria y no te digo siempre, pero en muchos casos, por lo menos en filosofía. (Charlotte Badger, entrevista personal, junio de 2016)

Estas formas de resistencia, al mismo tiempo, reproducen estereotipos y roles patriarcales y neoliberales. De modo que hace alusión a que sólo las mujeres responden desde lo emocional, lo generoso y lo solidario; y que el cuidado de la familia y sus criaturas depende cien por ciento de ellas. El tejer estas redes de cuidado, desafortunadamente contribuye a los objetivos de reproducir la auto explotación propia de la academia neoliberal. Vemos nuevamente la tensión y los costos de habitar la academia. Si ella desea permanecer, deberá asumir que el sistema no le dará ningún soporte, debe crear sus propias redes y con ello estará al servicio del sistema. Una paradoja en sí misma.

Es importante mencionar, que al igual que las estrategias de masculinización y malabarismo, la sororidad de académicas se construye para adaptarse y poder responder en base al "ideal de sujeto académico" que suele tener pocas o ninguna responsabilidad fuera del trabajo productivo (Acker, 1990).

\section{CONCLUSIONES}

En este escrito describimos la academia contemporánea regida por lógicas patriarcales y neoliberales. Presentamos cinco estrategias que las mujeres han tenido que crear o producir para poder habitar ese espacio académico: masculinización, perfeccionismo, malabarismo, limitación y sororidad. 
Consideramos importante categorizar y críticamente describir las estrategias, pues ayuda a visibilizar y describir las lógicas de opresión de los sistemas neoliberales y patriarcales. Las estrategias logran en definitiva que las mujeres (y no todas) entren y/o pertenezcan a la academia. Sin embargo, como hemos visto en cada una de las estrategias, se reproduce, ya sean las lógicas patriarcales o neoliberales

La categorización de las estrategias es didáctica, ya que estas pueden operar de manera simultánea en la cotidianeidad, en el análisis damos cuenta de esos momentos de superposición.

Cada una de estas estrategias, a su vez, proviene de las mujeres académicas y no como respuesta o alternativa del sistema. Es decir, las académicas son las que han tenido que dar solución a los problemas que la academia presenta y por tanto no ha habido un foco en el cuestionamiento de la academia como espacio opresor. Este es un dilema llamado double bind, donde, en cualquier escenario, la mujer académica se encontrará en una posición de desventaja (Seikkula y Olson, 2003) y el sistema continuará su opresión. Es decir, si la mujer académica se sigue valiendo de esas estrategias, es su responsabilidad y aunado a ello está reproduciendo alguna de las dos lógicas de opresión analizadas en este trabajo. Por el contrario, si la mujer académica decide no seguir con estas estrategias, corre el riesgo certero de no pertenecer a la academia. Vemos cómo la mujer académica está atrapada en una paradoja que pareciera ser que, independiente de cualquier estrategia que siga, el sistema ganará al continuar la opresión. Por lo tanto, este trabajo invita a un cambio de postura. A colocar el cuestionamiento no en las mujeres académicas, no en las estrategias de las que ellas se valen, sino en la denuncia de las lógicas de opresión neoliberales/patriarcales en la academia y reflexionar en torno a modificar las lógicas académicas contemporáneas.

En palabras de Chonchanok Viravan "If you see the glass ceiling, don't try to break it, try to change the building". "Si ves el techo de cristal, no trates de romperlo, trata de cambiar el edificio" (2007, 14 de mayo). Este trabajo no busca romper las estrategias, ni cuestionar/responsabilizar a las académicas sobre el uso de las estrategias. Busca el cuestionamiento urgente a la academia.

Nos preguntamos entonces ¿con qué otras lógicas (si existen) está operando la academia contemporánea?, ¿qué tipo de sujet@ produce? ¿Qué tan sostenible es producir sujet@s que deben crear estrategias de resistencia para poder incorporarse a la academia? ¿son la resistencia y reproducción las únicas alternativas? Y, finalmente, ¿es esta academia sostenible en el tiempo? 
En este trabajo nos enfocamos al sistema neoliberal/patriarcal, nos preguntamos sobre la intersección de otros marcadores de identidad como religión, raza o estatus migratorio. ¿Qué estrategias se han (hemos) necesitado para poder habitar la academia?

Sí, usamos nombres de mujeres piratas para hacer un homenaje a esas mujeres que lograron ser piratas, pese a tener todo en su contra (MacAlpine, 2016). No buscamos ratificar el triunfo de las lógicas individualistas neoliberales, sino de la acción. Nuestra posición consiste en problematizar el sistema patriar$\mathrm{cal} /$ neoliberal que oprime a las personas dentro de la academia, no a las personas que intentan sobrevivir en este medio. Las mujeres piratas siguen en el olvido, pocos son los documentos que versan sobre ellas (MacAlpine, 2016) pese a tener estrategias para habitar los mares y capitanear barcos. Sírvase este trabajo como una llamada de atención que, el hecho de tener estrategias para sobrevivir o habitar como las piratas, no garantiza la permanencia o el reconocimiento en el tiempo de las mujeres, por lo que debemos desmantelar "el edificio" en vez de cuestionar los mecanismos del mismo. Cuestionar a la academia en vez de responsabilizar a las mujeres que lo habitan.

A lo largo del análisis surgieron tres temas que consideramos deben ser explorados en próximas investigaciones. De especial interés para las autoras, es el tema de los afectos y emociones que las académicas nos narran como la inseguridad y culpa que aparecen frecuentemente, ya sea como causas o efectos de las estrategias que utilizan las académicas. En segundo lugar, la identidad de mujeres que son madres y habitan la academia. Si bien es una opresión que comparte algunos rasgos con la identidad mujer, no necesariamente se articula de la misma manera ya que las opresiones y estrategias de las que se valen cambiarían y urgen estudios al respecto. En tercer lugar, llama profundamente la atención que dentro de las entrevistas las académicas no hayan mencionado estrategias de autocuidado como tal, tema recurrente en el feminismo occidental, para poder sortear la academia y sus vidas.

Este trabajo tiene una deuda con las personas transgénero que habitan la academia, así como mujeres migrantes y personas pertenecientes a pueblos originarios. Es también una invitación a las personas lectoras a escribir e investigar al respecto. Confiamos que la lectura de nuestro trabajo inspire a seguir la lucha contra las políticas que actualmente rigen la academia y a develar otras formas de opresión y estrategias de sobrevivencia en la academia contemporánea. 


\section{REFERENCIAS}

Acker, Joan (1990). Hierarchies, jobs, bodies: A theory of gendered organizations. Gender \& Society, 4(2),139-58 https: / / doi.org/10.1177/089124390004002002

Amery, Fran; Bates, Stephen; Jenkins, Laura \& Savigny, Heather (2015). Metaphors on women in academia: A review of the literature, 2004-2013. Advances in Gender Research, 20, 247-267. https://doi.org/10.1108/S1529-212620150000020022

Appleby, John C. (2013). Women and English Piracy, 1540-1720. Boydell Press.

Berg, Maggie \& Seeber, Barbara (2016). Slow professor: Challenging the culture of speed in the academy. University of Toronto Press.

Bernasconi, Andres (2009). Gestión del cuerpo académico en las universidades chilenas: la institucionalización de la profesión académica en un contexto de mercado. Universidad Andrés Bello.

Biron, Caroline \& Karanika-Murray, María (2014). Process evaluation for organizational stress and well-being interventions: Implications for theory, method, and practice. International Journal of Stress Management, 21(1), 85.

https://doi.org/10.1037/a0033227

Blickenstaff, Jacob (2005). Women and science careers: Leaky pipeline or gender filter? Gender and Education, 17(4), 369-389. https://doi.org/10.1080/09540250500145072

Bravo Vargas, Viviana (2012). Neoliberalismo, protesta popular y transición en Chile, 1973-1989. Política y cultura, 37, 85-112. http://www.scielo.org.mx/scielo.php?script=sci_arttext\&pid=S0188$\underline{77422012000100005}$

Britton, Dana (2017). Beyond the Chilly Climate: The Salience of Gender in Women's Academic Careers. Gender and Society, 31(1), 5-27.

https://doi.org/10.1177/0891243216681494

Brunner, José (2008). Educación superior en Chile: instituciones, mercados y políticas gubernamentales. Leiden.

Cannella, Gaile. S. \& Salazar Perez, Michelle (2012). Emboldened Patriarchy in Higher Education: Feminist Readings of Capitalism, Violence, and Power. Cultural Studies Critical Methodologies, 12(4), 279-286. https://doi.org/10.1177/1532708612446421

Chandler, John; Barry, Jim \& Clark, Heather (2002). Stressing academe: The wear and tear of the new public management. Human Relations, 55(9), 1051-1069. https: / / doi.org/10.1177/0018726702055009019

CONICYT (2018). Compendio Estadístico CONICYT. https: / /www.conicyt.cl/wpcontent/uploads/2019/11/compendio-estadistico-CONICYT-2018.pdf

Cordingly, David (1996). Under the Black Flag: The Romance and the Reality of Life Among the Pirates. Random House. Print.

Denzin, Norman (2001). The reflexive interview and a performative social science. Qualitative Research, 1(1), 23-46. https://doi.org/10.1177/146879410100100102

Denzin, Norman \& Lincoln, Yvonna (2003). Strategies for qualitative inquiry. Sage. 
Durán Heras, María de los Ángeles (1981). Liberación y utopía: La mujer ante la ciencia. Akal. https://digital.csic.es/handle/10261/98367

Elson, Diane (2000). Progress of the world's women. UNIFEM Biennial Report, United Nations Development Fund for Women.

Enciso, Giazú \& Lara, Alí (2014). Emociones y ciencias sociales en el s. XX: La precuela del giro afectivo. Athenea Digital Journal, 14(1), 263-288.

https: / / doi.org/10.5565/rev/athenead/v14n1.1094

Fardella Cisternas, Carla (2013). Resistencias cotidianas en torno a la institucionalización del modelo neoliberal en las políticas educacionales: El caso de la docencia en Chile. Psicoperspectivas, 12(2), 83-92. https://doi.org/10.5027/psicoperspectivasVol12-Issue2-fulltext-294

Fardella Cisternas, Carla (2020). Abrir la jaula de oro. La universidad managerial y sus sujetos. Izquierdas, 49, 2299-2320.

Fardella Cisternas, Carla \& Corvalán Navia, Alejandra (2020). El tiempo en el conflicto trabajo-vida: El caso de las académicas en la universidad managerial. Psicoperspectivas, 19(3). https://doi.org/10.5027/ psicoperspectivas-vol19-issue3-fulltext-2051

Fardella Cisternas, Carla; Corvalán Navia, Alejandra \& Zavala Villegas, Ricardo (2019). El académico cuantificado. La gestión performativa a través de los instrumentos de medición en la ciencia. Psicología, Conocimiento y Sociedad, 9(2), 62-78. https://doi.org/10.26864/pcs.v9.n2.15

Fardella Cisternas, Carla; Sisto Campos, Vicente \& Jiménez Vargas, Felipe (2015). Nosotros los académicos. Narrativas identitarias y autodefinición en la universidad actual. Universitas Psychologica, 14(SPE5), 1625-1636.

https://doi.org/10.11144/Javeriana.upsy14-5.nani

Fardella Cisternas, Carla; Sisto Campos, Vicente y Jiménez Vargas, Felipe (2017). La transformación de la universidad y los dispositivos de cuantificación. Estudos de Psicologia, 34(3), 435-448. https://doi.org/10.1590/1982-02752017000300011

Gill, Rosalind (2009). Breaking the silence: The hidden injuries of neo-liberal academia. En Róisín Flood \& Rosalind Gill (Eds.), Secrecy and Silence in the Research Process: Feminist Reflections (pp. 1-21). Routledge. https: / /www.degruyter.com/downloadpdf/j/fs.2016.34.issue-1/fs-2016-0105/fs2016-0105.pdf

Glaser, Barney \& Straus, Anselm (1967). The Discovery of Grounded Theory: strategies for qualitative research. Aldine.

Gómez, Lucía; Jodar, Francisco \& Bravo, María (2015). Gubernamentalidad neoliberal y producción de conocimiento en la universidad: genealogía de una configuración subjetiva. Universitas Psychologica, 14(5), 1735-1750.

https://doi.org/10.11144/Javeriana.upsy14-5.gnpc

González Suárez, Mirta (2002). Feminismo, academia y cambio social. Revista Educación, 26(2),169-183. https://doi.org/10.15517/ revedu.v26i2.2913

Heijstra, Thamar; Steinthorsdóttir, Finnborg \& Einarsdóttir, Thorgerdur (2016). Academic career making and the double-edged role of academic housework. Gender and Education,29(6), 764-780 https://doi.org/10.1080/09540253.2016.1171825 
Heward, Christine (1994). Academic Snakes and Merit Ladders: Reconceptualising the 'glass ceiling'. Gender and Education, 6(3), 249-262.

https: //doi.org/10.1080/0954025940060302

Holstein, James \& Gubrium, Jaber (1995). The active interview. Sage. https://doi.org/10.4135/9781412986120

Lagarde, Marcela (2009, 11 de junio). La política feminista de la sororidad. Mujeres en Red, el periódico feminista. https: / / www.mujeresenred.net/spip.php?article1771

Lorber, Judith. (2008). Paradoxes of gender. Yale University Press.

MacAlpine, Anna (2016). Weather the Storm: Female Pirates, Sexual Diversity and the Reconstruction of Women's History. Tesis de maestría sin publicar. University of Calgary. https://doi.org/10.11575/PRISM/28680

Macfarlane, Bruce \& Burg, Damon (2019). Women professors and the academic housework trap. Journal of Higher Education Policy and Management, 41(3), 262-274. https://doi.org/10.1080/1360080X.2019.1589682

Manfredi, Simonetta; Grisoni, Louise; Handley, Karen; Nestor, Rebecca. \& Cooke, Felicity. (2014). Gender and higher education leadership: Researching the careers of top management programme alumni. Leadership Foundation for Higher Education.

Menzies, Heather \& Newson, Janice (2007). No Time to Think: Academics' life in the globally wired university. Time \& Society, 16(83), 82-98.

https://doi.org/10.1177/0961463X07074103

Merton, Robert (1973). The sociology of science: Theoretical and empirical investigations. University of Chicago Press.

Morales Casetti, Marjorie (2014). Nueva gestión pública en Chile. Revista de ciencia política (Santiago), 34(2), 417-438. https://doi.org/10.4067/S0718$\underline{090 \times 2014000200004}$

Oliva, María Angélica (2008). Política educativa y profundización de la desigualdad en Chile. Estudios pedagógicos (Valdivia), 34(2), 207-226. http://dx.doi.org/10.4067/S0718-07052008000200013

Ordorika, Imanol \& Navarro Minas (2006). Investigación académica y políticas públicas en la educación superior: El caso mexicano de pago por méritos. Revista del CEDES, 1, 52-71.

Oyarzún Maldonado, Cristian \& Cornejo Chávez, Rordrigo (2020). Teaching work and new public management in chile: a review of the evidence. Educação \& Sociedade, 41. https://doi.org/10.1590/ES.219509

Pujal, Margot (1994). La marca del género en la encrucijada entre subjetividad e intersubjetividad. Iztapalapa. Revista de Ciencias Sociales y Humanidades, 15(35), 131-142.

Pusser, Brian (2008). The state, the market and the institutional estate: Revisiting contemporary authority relations in higher education. In Michael, Paulsen (Ed.), Higher Education: Handbook of Theory and Research (pp. 105-139). Springer Netherlands. 
Ramos Claudio (2012a). Estructuras de comunicación en el campo de la ciencia social en Chile: un Análisis de Redes. Redes: revista hispana para el análisis de redes sociales, 23, 7-42. https://doi.org/10.5565/rev/redes.438

Ramos, Claudio (2012b). El ensamblaje de ciencia social y sociedad: Conocimiento científico, gobiernos de las conductas y producción de lo social. Ediciones Universidad Alberto Hurtado.

Salinas Ibacache, Carolina. (2019). El ingreso de la mujer a la Educación Superior en Chile desde sus orígenes a partir de la Promulgación del Decreto de Amunátegui en 1877 y su vinculación al Espacio Público en la primera década del S. XX. Tesis de doctorado sin publicar. http://repositorio.unab.cl/xmlui/handle/ria/10800

Santos, José (2015). Saberes acadêmicos: da produção textual à criação de conhecimento. Literatura: Teoría, Historia, Crítica, 17(2), 97-112.

https://doi.org/10.15446/lthc.v17n2.51276

Schiebinger, Londa (2008), Getting More Women into Science and Engineering Knowledge Issues. En Londa Schiebinger (Ed.), Gendered Innovations in Science and Engineering (pp. 365-378). Stanford University Press.

Seikkula, Jaakko \& Olson, Mary (2003). The open dialogue approach to acute psychosis: Its poetics and micropolitics. Family Process, 42(3), 403-418.

https://doi.org/10.1111/j.1545-5300.2003.00403.x

Sharma, Kumud (2003). Institutionalising Feminist Agenda(s). Economic and Political Weekly, 38(43), 4564-4566. https://www.jstor.org/stable/4414190

SIES (2018). Informe matrícula 2019 en Educación Superior en Chile. http://bibliotecadigital.mineduc.cl//handle/20.500.12365/4608

SIES (2021). Personal Académico en Educación Superior 2020. https://www.mifuturo.cl/wp-content/uploads/2021/01/Personal-Academico-eneducacion-superior_2020_SIES.pdf

Slaughter, Sheila \& Leslie, Larry (1997). Academic capitalism: Politics, policies, and the entrepreneurial university. The Johns Hopkins University Press.

Stanley, Jo (1995). Bold in Her Breeches: Women Pirates Across the Ages. Pandora, Print.

Tsouroufli, María (2018). Gendered and classed performances of 'good' mother and academic in Greece. European Journal of Women's Studies, 27(1), 9-24. https://doi.org/10.1177/1350506818802454

Tünnermann, Carlos \& De Souza, Mariela (2003). Desafíos de la universidad en la sociedad del conocimiento. Cinco años después de la conferencia mundial sobre Educación Superior. Revista Paper Series, 4(5), 1-28.

UNESCO (2000). La ciencia para el siglo XXI. Un nuevo compromiso. Conferencia Mundial sobre la Ciencia. Autor.

UNESCO (2017). Women in science. Fact Sheet No. 43. UNESCO Institute for Statistics. http: / / uis.unesco.org/sites/default/files/documents/fs43-women-in-science-2017$\underline{\text { en.pdf }}$ 
UNESCO (2019). Women un Science - 2019.

http://uis.unesco.org/sites/default/files/documents/fs55-women-in-science-2019$\underline{\text { en.pdf }}$

Viravan, Chonchanok (2007, 14 de mayo). Discurso principal Bussines and Professional Women International Conference [conferencia]. Seoul, Korea.

Wheelwright, Julie (1989). Women Who Dressed as Men in Pursuit of Life, Liberty and Happiness. Pandora Press.

\section{GIAZÚ ENCISO DOMÍNGUEZ}

Assistant Professor en la University of Houston Clear Lake. Dr. Enciso tiene un Ph.D. en Critical Social Psychology de la Universitat Autònoma de Barcelona. Sus intereses de investigación son el afecto y la emoción desde una perspectiva epistemológi$\mathrm{ca} /$ metodológica feminista. Fundadora/mentora del grupo de investigación Critical Interdisciplinary Studies conformado por estudiantes internacionales.

giazuenciso@uhcl.edu

https://orcid.org/0000-0003-2287-7666

\section{MAITE GONZÁLEZ-YÁÑEZ}

Profesor, ayudante y tutor en la Universidad Adolfo Ibáñez. Psicólogo y magister en psicología social de la Universidad Adolfo Ibañez. Sus intereses de investigación tienen relación con las políticas públicas, género y disidencias.

maite.begona.gonzalez@gmail.com

https://orcid.org/0000-0003-3832-1531

\section{FRANCESCA CHIAPPINI}

Psicóloga Clínica en Centro Interdisciplinario de las Mujeres. Psicóloga y magister en psicología social de la Universidad Adolfo Ibáñez. Sus intereses de investigación tienen relación a salud mental con perspectiva de género, sexualidad y mujeres en espacios masculinizados.

fchiappinik@gmail.com

https://orcid.org/0000-0001-5647-422X

\section{AGRADECIMIENTOS}

Agradecemos al FONDECYT Regular 1180129 "El trabajo científico en Chile" liderado por la investigadora Carla Fardella Cisternas. 


\section{FORMATO DE CITACIÓN}

Enciso Domínguez, Giazú, González-Yáñez, Maite \& Chiappini Koscina, Francesca (2021). Resistencias y reproducciones de mujeres académicas: Estrategias de supervivencia en la academia patriarcal/neoliberal. Quaderns de Psicologia, 23(2), e1603. https://doi.org/10.5565/rev/qpsicologia. 1603

HISTORIA EDITORIAL

Recibido: 19-01-2020

$1^{a}$ revisión: 25-02-2021

Aceptado: 3-08-2021

Publicado: 31-08-2021 